\title{
HIGHLIGHTS
}

NON-CODING RNA

\section{How plants silence stress}

Small interfering RNAs (siRNAs)

regulate gene expression by

the production

of endogenous

22-nt siRNAs

... might be

an important

mechanism

that enables

plants to

respond and

adapt to

environmental

abiotic stresses

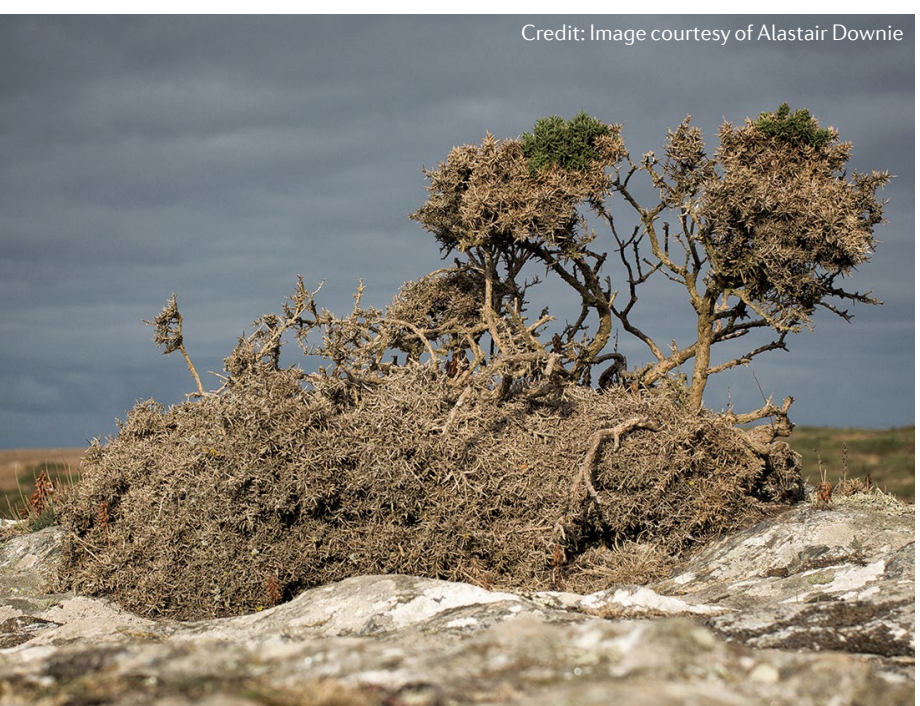

22-nt siRNAs, the authors went on to investigate whether DCL2 might also be involved in the production of endogenous 22-nt siRNAs.

They analysed plants carrying mutations in genes encoding RNA-degrading enzymes that would normally prevent the accumulation of mRNA-derived siRNAs, in combination with $D C L$ mutations. Mutations in these enzymes resulted in various developmental defects that were rescued by mutations in DCL2, suggesting that the developmental defects are cause by the accumulation of DCL2-dependent 22-nt siRNAs. Indeed, small-RNA sequencing confirmed an increase in the abundance of 22-nt siRNAs in the developmental mutants. When analysing the sequences of the $>1,100$ protein-coding genes from which these 22-nt siRNAs were produced, the authors found several genes involved in plant development, but, strikingly, two genes encoding nitrate reductases, NIA1 and NIA2, contributed respectively $45 \%$ and $48 \%$ of the total amount of 22 -nt siRNAs that accumulated in the plants with developmental defects.

Next, the authors investigated the mechanisms by which the 22-nt siRNAs might affect gene expression. Transcriptome profiling revealed that the mRNA levels of the genes that produce them were unaffected, but the NIA1 and NIA2 proteins were hardly detectable, even when proteasome-mediated degradation was inhibited, indicating that 22-nt siRNAs function by repressing translation. Moreover, the total amount of polysome-associated mRNA was reduced, suggesting global translation repression in addition to gene-specific translation repression. Translation repression was further confirmed using an in vitro cell-free system and by the finding that these 22-nt siRNAs require Argonaute 1 to function, which is a protein that associates with siRNAs to repress translation.

The growth and development defects associated with the accumulation of 22-nt siRNAs included reduction in root and seedling growth; alteration of phloem development; anthocyanin accumulation (as also seen when nitrogen metabolism is altered); reduction of photosynthesis and cell division; and activation of many stress response genes, together suggesting that 22-nt siRNAs coordinate plant growth and stress responses.

In wild-type plants, 22-nt siRNAs accumulated in conditions of nitrogen deficiency. Consistent with previous observations, NIA1 and NIA2 protein expression and mRNA polysome association were reduced. As amino acid synthesis relies heavily on NIA1/2-dependent and nitrite-reductase-dependent ammonium production, the authors suggest that the global translation repression might be, at least in part, caused by a shortage of amino acids. Thus, 22-nt siRNAs seem to be produced in response to nitrogen deficiency to dampen global translation and reduce plant growth. Importantly, 22-nt siRNAs also accumulated following treatment with abscisic acid (a plant hormone that regulates development and environmental stress responses) and in conditions of high salinity.

This study indicates that the production of endogenous 22-nt siRNAs, especially from the NIA1 and NIA2 genes, might be an important mechanism that enables plants to respond and adapt to environmental abiotic stresses, by reducing growth and activating defence mechanisms.

Kim Baumann

ORIGINAL ARTICLE Wu, H. et al. Plant 22-nt siRNAs mediate translational repression and stress adaptation. Nature https://doi.org/10.1038/ 41586-020-2231-y (2020) RELATED ARTICLE Borges, F. \& Martienssen, R. The expanding world of small RNAs in plants. Nat. Rev. Mol. Cell Biol. 16, 727-741 (2015) 